\title{
Des récepteurs à activité de phosphoprotéine phosphatase : une nouvelle classe de molécules
}

\section{Les nouvelles de ce numéro ont été préparées par Jean-Claude Dreyfus Axel Kahn Marc Peschanski}

1. Hunter T. Protein-tyrosine phosphatases: the other side of the coin. Cell 1989; $58: 1013$ 6.

2. Fagard R, Danielian S. Rôle des tyrosine protéine kinases dans l'activation des lymphocytes T. médecine/sciences 1989 ; 5 : 552 8.

$\mathrm{m} / \mathrm{s} n^{\circ} 10$ vol. 5 , décembre 89
Les récepteurs comportant un segment intracytoplasmique doté d'une activité de tyrosine kinase sont nombreux ; citons ceux de l'insuline, de l'IGF-1 (insulin-like growth factor type 1), des facteurs de croissance EGF, PDGF, CSF-1, etc. Plusieurs protéines solubles à activité de phosphotyrosine protéine phosphatase, catalysant la déphosphorylation des protéines à phosphotyrosine (par hydrolyse de la liaison phosphotyrosine) ont été plus récemment caractérisées [1]. L'une d'entre elles s'est révélée posséder un élément de séquence similaire à une région de la protéine CD45, un marqueur de la membrane leucocytaire, notamment des lymphocytes $T$. Cette protéine possède deux grandes régions hydrophiles, extra- et intracellulaire, séparées par un segment intramembranaire unique; elle ressemble donc étrangement à un récepteur dont l'occupation par un ligand encore inconnu modulerait l'activité phosphatasique intracellulaire; une telle activité a par ailleurs pu être démontrée expérimentalement.

Grâce au criblage à faible stringence de banques d'ADNc à l'aide d'une sonde spécifique de CD45, une molécule du même type a été identifiée, dénommée LAR. Si les domaines intracytoplasmiques de CD45 et de LAR se ressemblent, il n'en va pas du tout de même des domaines extracellulaires. Celui de LAR a de grandes similitudes avec la protéine N-CAM $\left(m / s n^{\circ} 2\right.$, vol. 4, p. 115), qui est exprimée à la membrane des cellules nerveuses et est responsable d'interactions spécifiques entre cellules syn- thétisant cette protéine. On peut donc faire l'hypothèse que, dans le cas de LAR, c'est l'interaction intercellulaire qui modulerait l'activité protéine phosphatasique du récepteur. Les mois qui viennent nous indiqueront si la diversité des récepteurs/protéine phosphatase est aussi grande que celle des récepteurs/protéine kinase, si les cibles cellulaires sont spécifiques ou non, si la liaison du ligand stimule ou inhibe l'activité de phosphoprotéine phosphatase, et enfin quels sont les ligands de ces nouveaux récepteurs.

L'étude de CD45 a permis d'apporter de premiers éléments de.réponse à ces questions: sa stimulation par des anticorps lorsqu'elle est liée au complexe CD3 ou à CD2 (protéines de transduction du signal d'activation des lymphocytes $T$ ) inhibe l'activation lymphocytaire, assez probablement par l'intermédiaire de la déphosphorylation de tyrosines impliquées dans la transmission du signal. En revanche, liée au complexe CD4/p56 lck, la CD45 stimulée déphosphorylerait la phosphotyrosine 505 de p56 $6^{\text {lck }}$, la désinhibant ainsi, ce qui aboutirait au déclenchement des phénomènes d'activation [2]. A noter que de telles protéines, capables de s'opposer aux oncogènes/tyrosine kinases en déphosphorylant leurs cibles, peuvent être considérées comme des antioncogènes dont l'altération homozygote pourrait, en théorie, conduire à la cancérisation cellulaire.

A. $\mathbf{K}$.
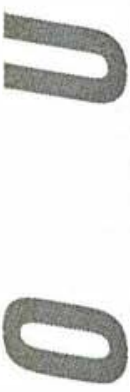

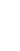

